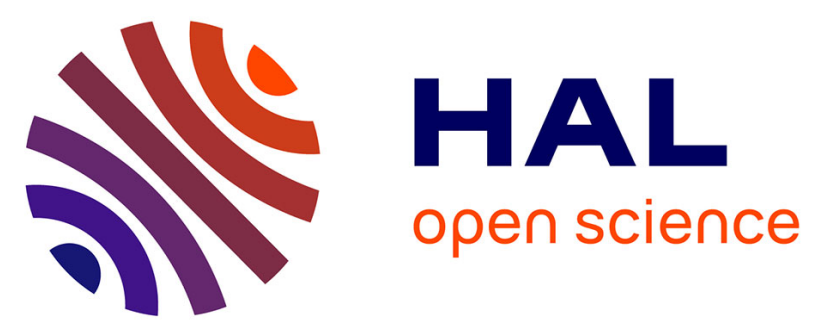

\title{
Influence of dry grinding in a ball mill on the length of multiwalled carbon nanotubes and their dispersion and percolation behaviour in melt mixed polycarbonate composites
}

Beate Krause, Tobias Villmow, Regine Boldt, Mandy Mende, Gudrun Petzold,

Petra Pötschke

\section{To cite this version:}

Beate Krause, Tobias Villmow, Regine Boldt, Mandy Mende, Gudrun Petzold, et al.. Influence of dry grinding in a ball mill on the length of multiwalled carbon nanotubes and their dispersion and percolation behaviour in melt mixed polycarbonate composites. Composites Science and Technology, 2011, 71 (8), pp.1145. 10.1016/j.compscitech.2011.04.004 . hal-00753181

\author{
HAL Id: hal-00753181 \\ https://hal.science/hal-00753181
}

Submitted on 18 Nov 2012

HAL is a multi-disciplinary open access archive for the deposit and dissemination of scientific research documents, whether they are published or not. The documents may come from teaching and research institutions in France or abroad, or from public or private research centers.
L'archive ouverte pluridisciplinaire HAL, est destinée au dépôt et à la diffusion de documents scientifiques de niveau recherche, publiés ou non, émanant des établissements d'enseignement et de recherche français ou étrangers, des laboratoires publics ou privés. 


\section{Accepted Manuscript}

Influence of dry grinding in a ball mill on the length of multiwalled carbon nanotubes and their dispersion and percolation behaviour in melt mixed polycarbonate composites

Beate Krause, Tobias Villmow, Regine Boldt, Mandy Mende, Gudrun Petzold, Petra Pötschke

PII: S0266-3538(11)00136-9

DOI: 10.1016/j.compscitech.2011.04.004

Reference: CSTE 4964

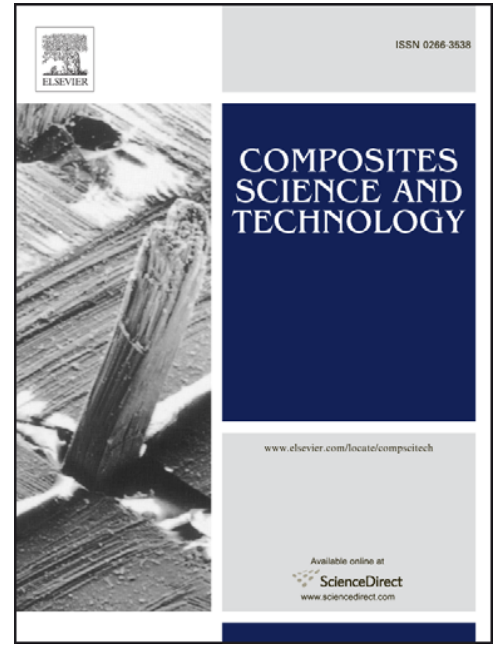

To appear in:

Composites Science and Technology

Received Date:

21 January 2011

Revised Date:

4 April 2011

Accepted Date:

7 April 2011

Please cite this article as: Krause, B., Villmow, T., Boldt, R., Mende, M., Petzold, G., Pötschke, P., Influence of dry grinding in a ball mill on the length of multiwalled carbon nanotubes and their dispersion and percolation behaviour in melt mixed polycarbonate composites, Composites Science and Technology(2011), doi: 10.1016/j.compscitech. 2011.04.004

This is a PDF file of an unedited manuscript that has been accepted for publication. As a service to our customers we are providing this early version of the manuscript. The manuscript will undergo copyediting, typesetting, and review of the resulting proof before it is published in its final form. Please note that during the production process errors may be discovered which could affect the content, and all legal disclaimers that apply to the journal pertain. 


\section{Influence of dry grinding in a ball mill on the length of multiwalled carbon nanotubes and} their dispersion and percolation behaviour in melt mixed polycarbonate composites

Beate Krause, Tobias Villmow, Regine Boldt, Mandy Mende, Gudrun Petzold, Petra Pötschke* Leibniz Institute of Polymer Research Dresden (IPF), Hohe Str. 6, 01069 Dresden, Germany

\section{Abstract}

Ball milling of carbon nanotubes (CNTs) in the dry state is a common way to produce tailored CNT materials for composite applications, especially to adjust nanotube lengths. For Nanocyl ${ }^{\mathrm{TM}}$ NC7000 nanotube material before and after milling for 5 and 10 hours the length distributions were quantified using TEM analysis, showing decreases of the mean length to 54 and $35 \%$, respectively. With increasing ball milling time in addition a decrease of agglomerate size and an increase of packing density took place resulting in a worse dispersability in aqueous surfactant solutions. In melt mixed CNT polycarbonate composites produced using masterbatch dilution step the electrical properties, the nanotube length distribution after processing, and the nano- and macrodispersion of the nanotubes were studied. The slight increase in the electrical percolation threshold in the melt mixed composites with ball milling time of CNTs can be assigned to lower nanotubes lengths as well as the worse dispersability of the ball milled nanotubes. After melt compounding, the mean CNT lengths were shortened to $31 \%, 50 \%$, and $66 \%$ of the initial lengths of NC7000, NC7000-5h, and NC7000-10h, respectively.

Keywords: A. Carbon nanotubes, A. Polymer-matrix composites (PMCs); B. Electrical properties; D. Transmission electron microscopy (TEM), Dispersion

"Corresponding author. Tel.: +49/3514658395; fax: +49/3514658565.

E-mail address: poe@ipfdd.de (P. Pötschke)

\section{Introduction}

Besides solution casting and in-situ polymerisation, melt mixing is a highly convenient method to produce thermoplastic based nanocomposites as it uses industrially available processing devices. In order to benefit from the outstanding intrinsic properties of carbon nanotubes (CNTs), a high degree of dispersion within the thermoplastic matrix is needed. This aim is generally hindered by 
the strong interactions between neighboured CNTs caused by high van-der-Waals forces and physical entanglements. The probability of entanglements depends thereby on the aspect ratio and the flexibility of the CNTs. Therefore, a reduction of entanglements due to a mechanical CNT shortening could improve their dispersability. One route towards mechanically shortened CNTs is the dry-state steel ball milling process which can help to tailor CNT properties [1,2]. CNTs are filled together with steel balls into a rotating barrel for the milling process resulting in the grinding of the CNT material to the necessary fineness by friction and impact with the tumbling balls. Some authors discuss the effect of ball milling on the nanotubes morphology [3-8]. Measurement of the nanotubes lengths using transmission electron microscopy (TEM) shows that nanotubes break and their length decrease in dependence on the ball milling time [1, 4-7]. Using X-ray Photoelectron Spectroscopy (XPS) measurements, an increase of oxygen proportion at long operation time was found caused by collision-induced cutting under ambient atmosphere introducing oxygen-containing functional groups to the cut nanotubes [4, 7]. Ahn et al. [8] described that the multiwalled carbon nanotubes (MWCNTs) tend to be compacted by impact of the balls and form agglomerates with increased size during the ball milling process as observed by scanning electron microscopy (SEM). The compactness or strength of CNT agglomerates influenced their dispersability. Krause et al. [9] described the dispersability of commercial MWCNT materials in aqueous surfactant solutions using centrifugal separation analysis. It was found that with higher agglomerate strength the dispersability of nanotubes worsened. Pegel et al. [10] described for two batches of the same MWCNTs different dispersabilities in aqueous surfactant solution. The incorporation of both kinds of MWCNTs in polycarbonate (PC) using melt mixing showed very different electrical percolation behaviours. The MWCNT material with the best dispersability leads to a significantly lower electrical percolation threshold in comparison to the MWCNTs with the worse dispersability. Comparable results were described from Krause et al. [11] for different kinds of CNTs and the electrical percolation behaviour of these CNTs in polyamide 6.6 and by Socher et al. [12] for composites based on polyamide 12. Nanotube materials with a good stability of their aqueous dispersions also showed a good dispersion after melt mixing as indicated by morphological investigations and exhibited low electrical percolation thresholds in the composites.

Concerning the length distribution of CNTs, some authors report measurements on pristine nanotubes using microscopic methods after dispersing nanotubes in suitable solvents or after 
dissolving them from a matrix. Albuerne et al. [13] dispersed pristine and functionalized multiwalled CNTs Baytubes ${ }^{\circledR} \mathrm{C} 150 \mathrm{P}$ in chloroform using ultrasound and determined the nanotubes length distribution using scanning force microscopy (SFM). SEM investigations on nanotube lengths after incorporation in an epoxy resins were reported by Fu et al. [14] whereby the epoxy matrix was dissolved using dimethylformamide (DMF) and low energy ultrasound. A significant CNT shortening from 10-20 $\mu \mathrm{m}$ up to $1.4 \mu \mathrm{m}$ was described. Chen et al. [15] measured the MWCNT length after poly(methyl methacrylate) composite preparation by solution-mixing using field-emission SEM. The MWCNT composites were dissolved in tetrahydrofuran (THF). The comparison between the as-grown and the processed MWCNT indicated a strong shortening of MWCNTs up to $90 \%$ of the initial length during the sonication assisted composite preparation after a nanotubes surface oxidation step. Duncan et al. [16] investigated the fragmentation aspect ratio of differently functionalized MWCNTs in PC composites prepared by a solvent precipitation method. For the fractured composites, PC was dissolved in THF and aspect ratio distributions of the partially broken nanotubes are shown. Lin et al. [17] determined the MWCNT lengths after melt dilution of a PC based masterbatch using SFM whereby a relatively harsh solvent and ultrasound treatment was used to extract the nanotubes from the PC matrix. Using different small scale mixers a comparable shortening of the nanotubes from $10-15 \mu \mathrm{m}$ to $0.4-0.6 \mu \mathrm{m}$ were measured. It has to be considered that ultrasonication of nanotube dispersions may lead to mechanical damage and shortening [18] changing the length to be studied. Krause et al. [19] performed SEM investigations on the remaining nanotubes after pyrolysis of small amounts of MWCNT-polyamide composites melt mixed in small-scale under different conditions. By this, changes in the nanotubes lengths after melt compounding using different rotation speed could be estimated.

A method to determine the CNT length of pristine CNTs and CNTs recovered from a composite using the same preparation and measurement method, namely by dispersing in chloroform, applying TEM on individualized nanotubes and performing image analysis, was recently described by Krause et al [20]. In this way the obtained results concerning the CNT length are comparable and CNT shortening during processing could be quantified.

In our study, as-grown and ball milled CNTs were characterized with regard to their morphology, nanotube length distribution, and dispersability in aqueous dispersion. In addition, these nanotubes were melt mixed in PC and the electrical percolation thresholds as well as the 
morphology using TEM and light microscopy (LM) of the composites were determined. Furthermore, the nanotubes were dissolved from the PC and the nanotube length distributions after melt mixing were measured. In this way, the shortening during melt mixing could be quantified.

\section{Experimental part}

\subsection{Materials}

This study is based on Nanocyl ${ }^{\mathrm{TM}}$ NC7000 (Nanocyl S.A., Sambreville, Belgium) MWCNT material, which was ball milled for 5 and 10 hours. Nanocyl ${ }^{\mathrm{TM}} \mathrm{NC7000}$ is an as-grown material produced in an industrial large-scale chemical vapour deposition process. It is characterized by an average nanotube diameter of $10 \pm 3 \mathrm{~nm}$ [21], a length of $1.5 \mu \mathrm{m}$, a carbon purity of $90 \%$, and a surface area of $250-300 \mathrm{~m}^{2} / \mathrm{g}$ [22]. The Nanocyl ${ }^{\mathrm{TM}} \mathrm{NC} 7000$ material was ball milled using a 5 litre jar half filled with stainless steel beads of $12 \mathrm{~mm}$ diameter. The remaining volume was filled with CNTs and the jar was rotated on two rubber rollers at a rotation speed of 50 revolutions per minute (rpm) for 5 (NC7000-5h) and 10 hours (NC7000-10h). The composites were processed by diluting a masterbatch containing $7.5 \mathrm{wt} . \%$ MWCNTs (Nanocyl $^{\mathrm{TM}}$ NC7000, NC7000-5h, or NC7000-10h) in PC (Lexan ${ }^{\mathrm{TM}}$ 141R, Sabic Innovative Plastics, The Netherlands) towards composites with different MWCNT contents. Both processing steps were performed using a co-rotating twin-screw extruder ZE25 (Berstorff, Germany) at a mean barrel temperature of $260^{\circ} \mathrm{C}, 500 \mathrm{rpm}$, a throughput of $5 \mathrm{~kg} / \mathrm{h}$ and an optimized screw configuration (SC5 in [23]). For the masterbatch production PC pellets and the powdery MWCNT material were fed simultaneously into the hopper by gravimetric dosing. For the masterbatch dilution, pellets of the masterbatch and the diluting PC were premixed.

\subsection{Methods}

\subsubsection{Characterization of dry MWCNT powder}

SEM of the MWCNT powders was performed using an Ultra plus microscope (Carl Zeiss GmbH, Oberkochen, Germany) on the as received material.

The agglomerate size distribution of the MWCNT powders was determined by laser diffraction using a Helos/BF particle size analyzer coupled with a RODOS dry dispersion unit and ASPIROS micro dose module (Sympatec GmbH, Clausthal-Zellerfeld, Germany). For the sample 
measurement a pressure of 0.5 bar was used. The measurement range is $4.5-875 \mu \mathrm{m}$. The volume weighted agglomerate size distributions were calculated in accordance to ISO 13320 combined with the Fraunhofer diffraction analysis [24-26]. Additionally, the parameters $\mathrm{x}_{10}, \mathrm{x}_{50}$, and $\mathrm{x}_{90}$ were calculated, indicating that $10 \%, 50 \%$, and $90 \%$ of the particles are lower than the given value.

All MWCNT materials were characterized by RAMAN spectroscopy using a Bruker FT-Raman spectrometer (excitation at $1064 \mathrm{~nm}$ ). In order to assess the concentration of defects of multiwalled carbon nanotubes the area ratio D/G between the disorder band at $1284 \mathrm{~cm}^{-1}$ (D-band) and the band at $1609 \mathrm{~cm}^{-1}$ (G-band) assigned to the in-plane vibrations of the graphitic walls was analysed.

XPS studies were performed on small powder samples using an AXIS ULTRA system (Kratos Analytical, UK) combined with a Mono-Al $\mathrm{K}_{1,2} \mathrm{X}$-ray-Source (300 W at $20 \mathrm{~mA}$ ) and an analysator having a pass energy of $160 \mathrm{eV}$ or $20 \mathrm{eV}$ was used.

\subsubsection{Preparation and characterization of aqueous MWCNT dispersions containing}

\section{surfactants}

To compare the dispersability of the three different kinds of MWCNTs the nanotubes were dispersed in an aqueous surfactant solution. According to previous investigations [11], the anionic surfactant sodium dodecyl benzene sulfonate (SDDBS) was used in this study. The carbon nanotubes were dispersed in a concentration of $0.07 \mathrm{~g} / \mathrm{l}$ in an aqueous solution of the SDDBS surfactant $(0.7 \mathrm{~g} / \mathrm{l})$. The formation of stable surfactant micelles is achieved since the concentration of SDDBS at $0.7 \mathrm{~g} / 1$ is above the critical micelle concentration of $0.42 \mathrm{~g} / 1$ [27]. The low concentration of CNTs was selected in order to ensure so-called swarm sedimentation of CNTs during the centrifugation step which means that the CNTs do not settle as a particle collective but

move according to their size [28]. CNT dispersions were prepared in a beaker glass at room temperature using an ultrasonic processor UP 200S (Hielscher Ultrasonics, Germany, $24 \mathrm{kHz}, 200$ W) equipped with a Sonotrode S14 made of titanium. The amplitude was adjusted to $20 \%$ resulting in a power output of $40 \mathrm{~W}$. The dispersion time was between 1 and 30 minutes to vary the energy input into the dispersions [9].

The sedimentation behaviour of the MWCNTs in aqueous dispersions containing surfactants was investigated by centrifugal separation analysis (CSA) using a LUMiSizer ${ }^{\circledR}$ LS611 (L.U.M. 
GmbH, Berlin, Germany). The LUMiSizer ${ }^{\circledR}$ is a microprocessor controlled analytical centrifuge that allows determining space and time resolved extinction profiles during centrifugation [28]. The centrifugation with high speed results in an accelerated sedimentation of particles. The CNT dispersions were filled into quadratic polystyrene tubes of $10 \times 10 \mathrm{~mm}^{2}$ and the evaluation of the transmission profiles as measured between the bottom and the fluid level allows the quantification of the dispersion stability. In case of good particle dispersion, all particles are wetted with surfactant and the stability of the dispersion is high, which corresponds with a low sedimentation rate. In case of bad dispersion remaining agglomerates settle fast. The LUMiSizer ${ }^{\circledR}$ experiments were carried out at $3000 \mathrm{rpm}$ for 45 minutes at room temperature. To evaluate the dispersion stability the integration of the transmission profiles was performed in the middle region of the tube between 106 and $124 \mathrm{~mm}$. The LUMiSizer ${ }^{\circledR}$ experiments are reproducible and the results are based on double measurements. Additionally, the intensity weighted particle size distribution of the dispersed MWCNTs was calculated from sedimentation velocity distribution according to ISO 13318 described in detail in [9]. As one result of the analysis the average mean value of particle diameter $\mathrm{x}_{50}$ was obtained.

\subsubsection{Characterization of MWCNTs filled PC composites}

The electrical volume resistivity of the composite materials was determined according to the standards ASTM D 4496 and ASTM D 257. Plates having diameters of $60 \mathrm{~mm}$ and a thickness of $0.5 \mathrm{~mm}$ were compression-moulded from the pelletised materials received from the extruder. The materials were hot pressed by means of a PW40 EH press (Paul-Otto Weber GmbH, Germany) at $260^{\circ} \mathrm{C}$ for 3 minutes applying a pressing speed of $6 \mathrm{~mm} / \mathrm{s}$. Subsequently the samples were quenched at $6^{\circ} \mathrm{C}$ between cooling plates.

The resistivity measurements were performed at room temperature on strips $\left(30 \times 0.5 \times 3 \mathrm{~mm}^{3}\right)$ cut from the plates using a 4-point Test Fixture (gold contacts with a distance of $16 \mathrm{~mm}$ between the source electrodes and $10 \mathrm{~mm}$ between the measuring electrodes) in combination with a Keithley multimeter Model 2000. The values higher than $10^{7} \Omega \mathrm{cm}$ were measured on the pressed plates using a Keithley 8009 Resistivity test fixture combined with a Keithley 6517A electrometer. All sample surfaces were cleaned with ethanol prior resistivity measurements. In order to get an estimate for the percolation concentration $p_{c}$ the experimental conductivity data $\sigma(p)$ were fitted for volume concentrations $p>p_{c}$ to equation: 
$\sigma(p)=B\left(p-p_{c}\right)^{t}$

which represents the well known scaling law for the composite conductivity near the electrical percolation threshold [29]. The values $B, p_{c}$ and $t$ were fitted using the method of the lowest value of the root mean square error. As CNT density a value of $1.75 \mathrm{~g} / \mathrm{cm}^{3}$ was used [30].

Light microscopy (LM) in transmission mode was performed to investigate the macrodispersion of CNT agglomerates within the PC matrix. Thin sections with a thickness of $20 \mu \mathrm{m}$ were cut from extruded strands using a microtome Leica 2055 (Leica Mikrosysteme Vertrieb GmbH, Bensheim, Germany) and were fixed with Entellan ${ }^{\circledR}$ (Merck KGaA, Darmstadt, Germany) on glass slides. The LM images were recorded using a microscope $\mathrm{BH} 2$ combined with a camera DP71 (Olympus, Deutschland GmbH, Hamburg, Germany). The images were analysed using the software SCANDIUM 5.1 (Olympus Soft Imaging Solutions GmbH, Münster, Germany). An analytical TEM (LIBRA120, Carl Zeiss GmbH, Jena, Germany) was used to investigate the state of CNT network formation depending on the ball milling process. The microscope was adjusted with an acceleration voltage of $120 \mathrm{kV}$, whereas the use of a zero loss filter led to best filler/matrix contrasts. Ultra-thin sections with a thickness of $80 \mathrm{~nm}$ were cut from extruded strands at room temperature using a Reichert Ultracut S ultramicrotome (Leica, Germany) in combination with a diamond knife (Diatome, Switzerland).

\subsubsection{Determination of the nanotube length distribution}

The investigations of the nanotube length distribution were performed using a TEM procedure developed by Krause et al. [20]. The MWCNT powders were dispersed in chloroform and PC composites containing $2 \mathrm{wt} \%$ MWCNTs were dissolved in chloroform for 1 hour. All dispersions were treated in an ultrasonic bath (SONOREX RK100H, Bandelin Electronic GmbH, Berlin, Germany, $35 \mathrm{kHz}$, ultrasonic power $80 \mathrm{~W}$ ) for 3 minutes. One drop of the dispersion was located on a TEM grid with a carbon coating having a tissue paper underneath ensuring the removal of solvent. In the TEM images collected with a Libra120 or a Libra200 (Carl Zeiss GmbH, Germany) the nanotube lengths were measured on approximately 400 nanotubes for each kind of CNT material. The software SCANDIUM 5.1 (Olympus Soft Imaging Solutions GmbH, Münster, Germany) was applied using the full visible length of each separated nanotube not touching the edge of the image by applying the polyline function. In order to measure the length of very long nanotubes in some cases several images were stitched together. The results are given as number 
distributions with class sizes of $100 \mathrm{~nm}$. Additionally, the parameters $\mathrm{x}_{10}, \mathrm{x}_{50}$, and $\mathrm{x}_{90}$ were calculated to characterize the distribution indicating that 10,50 , and $90 \%$ of the nanotubes are smaller than the given value.

\section{Results}

\subsection{Characterization of dry MWCNT powder}

In order to access the state of the primary agglomerates before incorporating in the matrix the different powders were observed by SEM (Figure 1). It was found that the size of primary agglomerates was reduced significantly after ball milling. In addition, for NC7000 a relatively loose structure was visible. The combed yarn structure as described in literature and clearly shown e.g. in [9] could be not seen at the used high magnification. As it is evident at low magnification, the ball milling results in more compact MWCNT agglomerates with higher packing density, as described earlier by Ahn et al. [8].

The primary CNT agglomerate size distributions determined by laser diffraction are given in Figure 2 as cumulative curves. The smallest agglomerates were found for both ball milled CNTs. All detected particles were smaller than $50 \mu \mathrm{m}$, however $10 \%$ of the NC7000-5h and $20 \%$ of NC7000-10h powder contained agglomerates smaller than the measurement range of 4.5-875 $\mu \mathrm{m}$. Significantly higher agglomerate sizes were determined for NC7000. The smallest agglomerates were about $675 \mu \mathrm{m}$ whereas only $12 \%$ of the particles were within the measurement range of the equipment up to $875 \mu \mathrm{m}$.

The CNT length distributions of the three different CNT materials and the $\mathrm{x}_{10}, \mathrm{x}_{50}$, and $\mathrm{x}_{90}$ values describing the distributions as determined using TEM are shown in Figure 3 including a TEM image of N7000-10h. The highest CNT length and a very broad distribution up to $6 \mu \mathrm{m}$ were found for the as-grown NC7000. The ball-milled CNTs were significantly shorter than the asgrown material, whereas the longer ball milling treatment leads to shorter CNTs. The width of the length distribution decreased with ball milling time. The $\mathrm{x}_{10}, \mathrm{x}_{50}$, and $\mathrm{x}_{90}$-values (Figure 3 ) of NC7000-5h are nearly halved as compared to NC7000. For NC7000-10h the $\mathrm{x}_{10}, \mathrm{x}_{50}$, and $\mathrm{x}_{90^{-}}$ values are one third as compared to the values of NC7000. The $\mathrm{x}_{50}$ values of NC7000-5h and NC7000-10h are 54\% and 35\% of the initial NC7000. The same trend was described by Kim et al. [3], who found a significant decrease of MWNT length with increasing ball milling time. 
RAMAN investigations were carried out to estimate the defect density of the different CNTs. A D/G area ratio of 2.2 was found for the as-grown CNTs and the CNTs ball milled for 5 hours. After 10 hours of ball milling the D/G-area ratio was slightly lower at 1.9. It can be assumed that there is no significant difference in the microstructure of the three kinds of CNTs. This finding is in agreement with Kim et al. [3] who found no big differences in the structure of as-grown and ball-milled CNTs as investigated by RAMAN experiments.

XPS studies revealed higher oxygen $(\mathrm{O})$ to carbon $(\mathrm{C})$ ratio for ball-milled CNTs, which can be considered as a higher functionalisation degree of the outer CNT wall. The O:C ratio was calculated to be 0.0045 (NC7000), 0.0117 (NC7000-5h), and 0.0156 for NC7000-10h. The increase of oxygen content with ball milling time was also described by Smart et al. [4] for singleand doublewalled CNT and Park et al. [7] for MWCNTs.

\subsection{Characterization of CNTs in aqueous surfactant dispersion}

The sedimentation behaviour of carbon nanotubes in the aqueous dispersion containing surfactant was investigated under centrifugation forces to estimate the dispersability of the different CNT materials. For all CNT dispersions horizontal transmission profiles in the CSA were observed caused by their very broad agglomerate size distributions (not shown here, see e.g. Fig. 1 in [11]) and all three dispersions showed decreasing values of integral transmission with ultrasonic treatment time (Figure 4). This is caused by the increasing number of individualized CNTs and very small agglomerate fragments. However, for the dispersion containing Nanocyl ${ }^{\mathrm{TM}} \mathrm{NC7000}$ the amount of dispersed nanotubes increased faster. Already after two minutes ultrasonic treatment stable dispersions were obtained indicated by a constant value of integral transmission. A significant longer ultrasonication time of 4 to $10 \mathrm{~min}$ was needed to get stable dispersions for the ball-milled CNTs. Summarizing, NC7000 shows the best dispersability in an aqueous surfactant dispersion in comparison to the ball milled CNTs. The ball milling treatment resulted in worse dispersability so that higher energy input is necessary to prepare stable dispersions resulting from compacted primary agglomerates having a higher packing density.

The intensity weighted particle size distributions of dispersed particles were determined from the CSA results. During CSA the particles are affected by centrifugal forces and shear stress whereby the particles are oriented in the sedimentation direction [31,32]. Figure 5 shows the average particle diameter $\mathrm{x}_{50}$ after 45 min of centrifugation in dependence on the ultrasonic treatment time. 
It was found that the particle size decreased exponentially with ultrasonic treatment time for all three kinds of CNTs whereas the particle sizes were quite different. An ultrasonic treatment of NC7000-5h for 4 min resulted in an average particle diameter $\mathrm{x}_{50}$, which is comparable to the value obtained for the untreated CNTs after $2 \mathrm{~min}$. To get a similar $\mathrm{x}_{50}$ value, for NC7000-10h fifteen min ultrasonication was needed. The results indicate that the ball milled CNT materials consist of primary agglomerates having a higher cohesive strength, as more energy is needed to disperse them. The reason for this finding is the compact primary CNT agglomerate structure resulting from the milling process. The van-der-Waals forces between neighboured CNTs are inversely proportional of their distance in the sixth power.

Summarizing the results from CSA, for the ball milled CNTs worse dispersability and larger dispersed particles were found in comparison to the as-grown NC7000 and the effects increased with ball milling time. For a good dispersion of the ball milled CNTs in aqueous surfactant solutions more energy input was needed than for as-grown NC7000.

\subsection{Electrical percolation behaviour of MWCNTs filled PC composites}

NC7000 and ball milled CNTs were incorporated in PC using twin-screw extrusion and electrical resistivity was measured on compression moulded plates. The electrical volume resistivities as a function of CNT content and kind of CNTs are shown in Figure 6. The electrical percolation threshold $p_{c}$, the exponent $t$ and the proportionality constant $B$ were fitted and summarized in a table in Figure 6. The electrical percolation threshold $p_{c}$ of PC based composites slightly rose with the ball milling treatment time of CNTs. The lowest electrical percolation threshold was found for PC filled with as received NC7000 at 0.175 vol.\% (0.25 wt.\%). Composites containing NC7000$5 \mathrm{~h}$ or NC7000-10h were electrically percolated at 0.23 vol.\% (0.33 wt.\%) or 0.26 vol.\% (0.38 wt.\%), respectively. The exponent $t$ was between 1.35 and 1.78. An exponent of 2 corresponds to the value applying the universal 3D lattice model [29]. The factor $B$ giving a measure for the maximum conductivity was similarly for the three different composites in the range of $0.01-$ $0.02 \mathrm{~S} / \mathrm{cm}$. In the literature $[34,35]$, for melt compounded PC-MWCNT composites an electrical percolation threshold of $0.5 \mathrm{wt} . \%$ was described for pressed plates.

\subsection{Macro- and nanodispersion of MWCNTs in PC composites}


The state of CNT dispersion within the PC based composites was investigated for composites containing 1 wt.\% CNTs by means of LM and TEM. LM images of thin sections cut from composites containing the different kinds of CNTs indicating the CNT macrodispersion are shown in Figure 7. The composite filled with NC7000 was free of primary agglomerates. In contrast, the polycarbonate containing ball milled CNTs included few small agglomerates visible as black dots whereas more agglomerates were found in the NC7000-10h filled composite. TEM images indicating the state of CNT dispersion of the extruded strands in nanoscale are shown in Figure 8. A relatively good dispersion but slightly inhomogeneous CNT distribution of individual CNTs exhibiting a slight clustering behaviour possibly induced by secondary agglomeration at the end of the extrusion process was found for all three PC/CNT composites. It can be assumed that these CNT clusters were part of the percolation network as these composites were electrically conductive.

\subsection{MWCNT length distribution after processing}

The MWCNT length distribution was determined using TEM in order to evaluate the influence of melt processing on the CNTs aspect ratio using PC composites containing 2 wt.\% MWCNTs. In comparison to the unprocessed CNT materials (Figure 3), the processed CNTs show a significant shortening and a decreased width of the length distributions resulting from the melt processing (Figure 9). The largest decrease of nanotube length was observed for NC7000, where the $\mathrm{x}_{50^{-}}$ value decreases from 1341 to $418 \mathrm{~nm}$. For N7000-5h or N7000-10h a reduction of the $\mathrm{x}_{50}$-value due to the melt processing from 726 to $366 \mathrm{~nm}$ or 466 to $309 \mathrm{~nm}$ was measured, respectively. As an example for the quantitative analysis of CNT lengths Figure 10 shows an original TEM image and the same image with marked lengths of the measured MWCNTs. Only completely visible nanotubes were used for the measurements. Assuming that the CNT diameter were not influenced by the ball milling, based on the $\mathrm{x}_{50}$-values a reduction of the aspect ratio to $31 \%, 50 \%$, and $66 \%$ can be estimated for NC7000, NC7000-5h, and NC7000-10h, respectively. The finding of nanotubes shortening after processing is in good agreement with results presented by Fu et al. [14] who described a significant CNT shortening after the incorporation into an epoxy matrix using a three-roll calendaring processing.

\section{Summary}


The influence of ball milling on the properties of nanotubes und their composites with $\mathrm{PC}$ was studied. The as-grown Nanocyl ${ }^{\mathrm{TM}}$ NC7000 material was treated for 5 and 10 hours in the dry state. With the analysis of the nanotubes lengths of dry CNTs and CNTs dissolves from the composites, for the first time a quantification of the shortening during ball milling process as well as during the melt compounding process was performed.

The pristine nanotubes powders were investigated by SEM and higher packing densities were found for the ball milled CNTs. The determination of volume weighted particle size distribution using laser diffraction showed a significant influence of the milling process on the agglomerate size of the as-grown material. Nanocyl ${ }^{\mathrm{TM}} \mathrm{NC7000}$ having agglomerate sizes mainly at over 675 $\mu \mathrm{m}$ were shorted to particle sizes lower than $50 \mu \mathrm{m}$ after ball milling. The investigations of the nanotube length distribution show a significant shortening of the CNTs after the ball milling process. Based on the $\mathrm{x}_{50}$-values a reduction of the nanotubes lengths to $54 \%$ and $35 \%$ can be estimated for NC7000-5h and NC7000-10h in comparison to as-grown NC7000. The D/G ratio calculated by RAMAN of all nanotubes was comparable and decreased only slightly for NC700010h. In the XPS study a higher [O]:[C] ratio for the ball milled nanotubes was found indicating the formation of oxygen groups on the nanotubes surface. The dispersability of nanotubes in an aqueous surfactant solution as studied using centrifugal separation analysis was significantly worsened for the ball milled CNTs as compared to the as-grown material. The worse dispersability of the ball milled nanotubes was also found in melt mixed samples. LM investigations on thin composite sections indicated for the PC composites filled with NC7000-5h or NC7000-10h few agglomerates whereas the PC/NC7000 composites were free of primary agglomerates. The composite preparation performed by dilution of a masterbatch is a process with relatively high energy input in the composite and is favourable to disperse nanotubes even with a worse dispersability. As a result of this processing procedure, in the TEM images of all composites separated nanotubes were visible.

The electrical behaviour of the melt mixed PC composites indicated that the electrical percolation threshold increased slightly with ball milling treatment time of the nanotubes. The formation of a conductive network in the composites depends on the properties of the nanotubes, e.g. aspect ratio and dispersability, and on the processing conditions (here masterbatch dilution). The investigation of the CNT powders showed that with ball milling treatment time the nanotubes length and thus the aspect ratio as well as the dispersability were decreased. For the CNTs extracted from the PC 
composites an additional decrease of the nanotubes length to $31-66 \%$ was found caused by the melt mixing process. However, even after melt processing NC7000 had higher nanotubes lengths than NC7000-5h and NC7000-10h. These findings are an explanation for the slightly lower electrical percolation threshold of the melt mixed composites with NC7000 in comparison to the ball milled nanotubes.

\section{Acknowledgement}

The authors thank Nanocyl S.A. (Belgium) for providing the ball milled nanotubes and Manuela Heber, Uwe Geißler and Bernd Kretzschmar (all IPF) for technical support.

\section{Figure chapter}

Fig. 1: SEM images of the pristine MWCNT powder at two magnifications: A) NC7000, B) NC7000-5h, and C) NC7000-10h.

Fig. 2: Cumulative volume weighted distribution of mean agglomerate size of the different dry MWCNT powders using laser diffraction method.

Fig. 3: Nanotube length distribution of the pristine and ball milled MWCNT powders using TEM (total number of particles: 314 (NC7000), 390 (NC7000-5h), 420 (NC7000-10h)) including a TEM image of NC7000-10h and characteristic length values.

Fig. 4: Integral transmission (after 45 minutes centrifugation time) in dependence on ultrasonic treatment time for different aqueous MWCNT dispersions using CSA.

Fig. 5: Average particle diameter $\mathrm{x}_{50}$ from intensity weighted particle size distribution determined by CSA in dependence on ultrasonic treatment time for different aqueous MWCNT dispersions. Fig. 6: Electrical volume conductivity of PC composites containing different MWCNTs in dependence on the MWCNT content including fit parameters.

Fig 7: Light transmission microscopy image of a PC composites containing $1 \mathrm{wt}$ \% MWCNT: A) NC7000, B) NC7000-5h, and C) NC7000-10h.

Fig 8: TEM image of a PC composites containing 1 wt.\% MWCNTs: A) NC7000, B) NC7000-5h, and C) NC7000-10h.

Fig. 9: Nanotube length distribution as obtained from TEM images of the processed CNTs dissolved from the composites with 2 wt.\% MWCNTs including characteristic length values (total number of particles: 409 (NC7000), 427 (NC7000-5h), 408 (NC7000-10h)). 
Fig 10: TEM images of the MWCNTs dissolved from the PC composites containing 2 wt.\% NC7000-5h: A) original image, B) image with exemplary MWCNTs length measurements.

\section{References}

[1] Pierard N, Fonseca A, Konya Z, Willems I, Van Tendeloo G, B.Nagy J. Production of short carbon nanotubes with open tips by ball milling. Chemical Physics Letters 2001;335(1-2):1-8.

[2] Salver-Disma F, Tarascon JM, Clinard C, Rouzaud JN. Transmission electron microscopy studies on carbon materials prepared by mechanical milling. Carbon 1999;37(12):1941-1959.

[3] Kim YA, Hayashi T, Fukai Y, Endo M, Yanagisawa T, Dresselhaus MS. Effect of ball milling on morphology of cup-stacked carbon nanotubes. Chemical Physics Letters 2002;355(3-4):279-284.

[4] Smart SK, Ren WC, Cheng HM, Lu GQ, Martin DJ. Shortened double-walled carbon nanotubes by high-energy ball milling. International Journal of Nanotechnology 2007;4(5):618-633.

[5] Chen L, Qu M-z, Zhou G-m, Zhang B-1, Yu Z-1. PC-mediated shortening of carbon nanotubes. Materials Letters 2004;58(29):3737-3740.

[6] Sun YF, Zhang AM, Yin Y, Dong YM, Cui YC, Zhang X, Hong JM. The investigation of adsorptive performance on modified multi-walled carbon nanotubes by mechanical ball milling. Materials Chemistry and Physics 2007;101(1):30-34.

[7] Park KC, Fujishige M, Takeuchi K, Arai S, Morimoto S, Endo M. Inter-collisional cutting of multi-walled carbon nanotubes by high-speed agitation. Journal of Physics and Chemistry of Solids 2008;69(10):2481-2486.

[8] Ahn JH, Shin HS, Kim YJ, Chung H. Structural modification of carbon nanotubes by various ball milling. Journal of Alloys and Compounds 2007;434-435:428-432.

[9] Krause B, Mende M, Pötschke P, Petzold G. Dispersability and particle size distribution of CNTs in an aqueous surfactant dispersion as a function of ultrasonic treatment time. Carbon 2010;48(10):2746-2754.

[10] Pegel S, Pötschke P, Petzold G, Alig I, Dudkin SM, Lellinger D. Dispersion, agglomeration, and network formation of multiwalled carbon nanotubes in polycarbonate melts. Polymer 2008;49(4):974-984.

[11] Krause B, Petzold G, Pegel S, Pötschke P. Correlation of carbon nanotube dispersability in aqueous surfactant solutions and polymers. Carbon 2009;47(3):602-612.

[12] Socher R, Krause B, Boldt R, Hermasch S, Wursche R, Pötschke P. Melt mixed nanocomposites of PA12 with MWNT: Influence of MWNT and matrix properties on macrodispersion and electrical properties. Composites Science and Technology 2011;71(3):306-314. 
[13] Albuerne J, Boschetti-de-Fierro A, Abetz V. Modification of multiwall carbon nanotubes by grafting from controlled polymerization of styrene: Effect of the characteristics of the nanotubes. Journal of Polymer Science Part B: Polymer Physics 2010;48(10):1035-1046.

[14] Fu S-Y, Chen Z-K, Hong S, Han CC. The reduction of carbon nanotube (CNT) length during the manufacture of CNT/polymer composites and a method to simultaneously determine the resulting CNT and interfacial strengths. Carbon 2009;47(14):3192-3200.

[15] Chen L, Ozisik R, Schadler LS. The influence of carbon nanotube aspect ratio on the foam morphology of MWNT/PMMA nanocomposite foams. Polymer 2010;51(11):2368-2375.

[16] Duncan RK, Chen XG, Bult JB, Brinson LC, Schadler LS. Measurement of the critical aspect ratio and interfacial shear strength in MWNT/polymer composites. Composites Science and Technology 2010;70(4):599-605.

[17] Lin B, Sundararaj U, Pötschke P. Melt mixing of polycarbonate with multi-walled carbon nanotubes in miniature mixers. Macromolecular Materials and Engineering 2006;291(3):227-238.

[18] Lu KL, Lago RM, Chen YK, Green MLH, Harris PJF, Tsang SC. Mechanical damage of carbon nanotubes by ultrasound. Carbon 1996;34(6):814-816.

[19] Krause B, Pötschke P, Häußler L. Influence of small scale melt mixing conditions on electrical resistivity of carbon nanotube-polyamide composites. Composites Science and Technology 2009;69(10):1505-1515.

[20] Krause B, Boldt R, Pötschke P. A method for determination of length distributions of multiwalled carbon nanotubes before and after melt processing. Carbon 2011;49(4):1243-1247.

[21] Tessonnier J-P, Rosenthal D, Hansen TW, Hess C, Schuster ME, Blume R, Girgsdies F, Pfänder N, Timpe O, Su DS, Schlög1 R. Analysis of the structure and chemical properties of some commercial carbon nanostructures. Carbon 2009;47(7):1779-1798.

[22] Nanocyl. Data sheet Nanocyl 7000. Edition 2007-02-05. Sambreville, Belgium; 2007.

[23] Villmow T, Kretzschmar B, Pötschke P. Influence of screw configuration, residence time, and specific mechanical energy in twin-screw extrusion of polycaprolactone/multi-walled carbon nanotube composites. Composites Science and Technology 2010;70(14):2045-2055.

[24] Yamamoto H, Matsuyama T, Wada M. Shape distinction of particulate materials by laser diffraction pattern analysis. Powder Technology 2002;122(2-3):205-211.

[25] Matsuyama T, Yamamoto H. Particle shape and laser diffraction: A discussion of the particle shape problem. Journal of Dispersion Science and Technology 2004;25(4):409-416.

[26] Heike M, Hirleman ED. Laser Diffraction Spectroscopy: Influence of Particle Shape and a Shape Adaptation Technique. Particle and Particle Systems Characterization 1998;15(4):163-169.

[27] Jönsson B, Lindman B, Holmberg K, Kronberg B. Surfactants and Polymers in Aqueous Solution. New York: John Wiley Sons, 1998.

[28] Lerche D. Dispersion stability and particle characterization by sedimentation kinetics in a centrifugal field. Journal of Dispersion Science and Technology 2002;23(5):699-709. 
[29] Stauffer D, Aharony A. Introduction in percolation theory. London: Taylor and Francis, 1994.

[30] Shaffer MSP, Windle AH. Fabrication and characterization of carbon nanotube/poly(vinyl alcohol) composites. Advanced Materials 1999;11(11):937-941.

[31] Henn AR. Calculation of the Stokes and Aerodynamic Equivalent Diameters of a short reinforcing fiber. Particle and Particle Systems Characterization 1996;13(4):249-253.

[32] Butler JE, Shaqfeh ESG. Dynamic simulations of the inhomogeneous sedimentation of rigid fibres. Journal of Fluid Mechanics 2002;468(1):205-237.

[33] Heaney MB. Measurement and interpretation of nonuniversal critical exponents in disordered conductor-insulator composites. Physical Review B 1995;52(17):12477.

[34] Pötschke P, Zschoerper NP, Moller BP, Vohrer U. Plasma Functionalization of Multiwalled Carbon Nanotube Bucky Papers and the Effect on Properties of Melt-Mixed Composites with Polycarbonate. Macromolecular Rapid Communications 2009;30(21):1828-1833.

[35] Pflug G, Reinemann S, Hungsberg A, Wipprecht A. Kunststoffgehäuse abschirmen. Kunststoffe 2008;(2):94-98. 

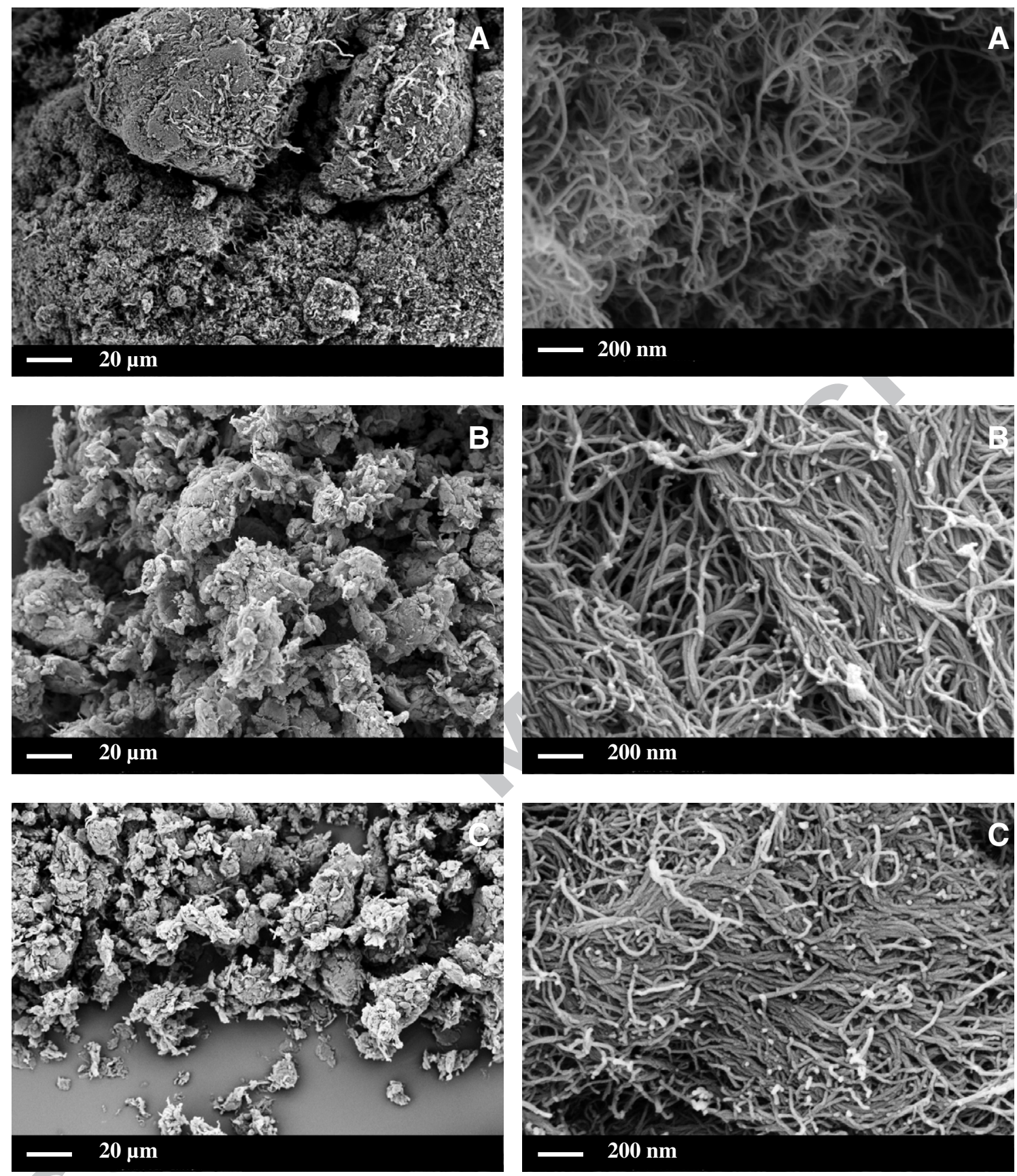

Fig. 1 


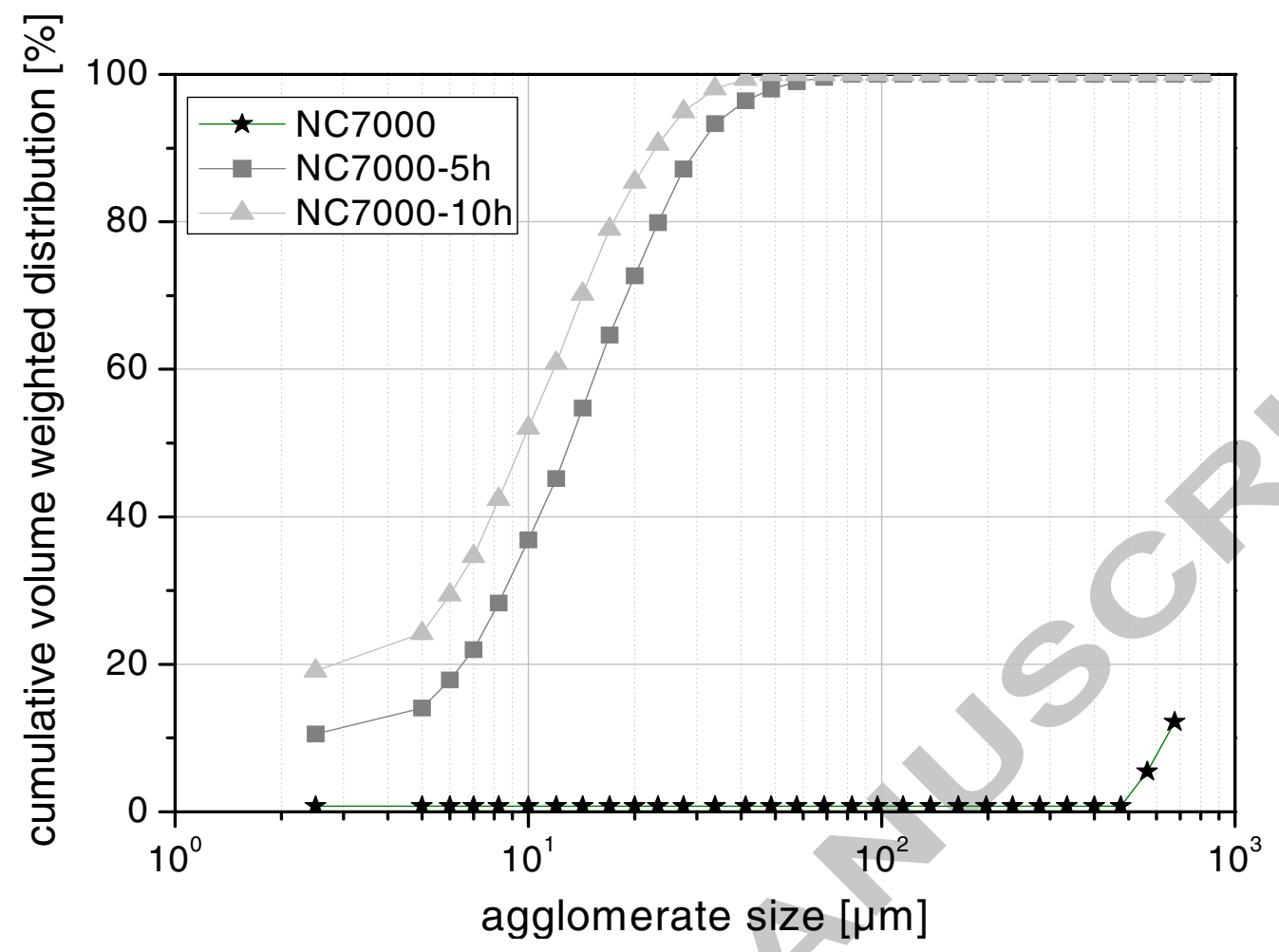

Fig. 2

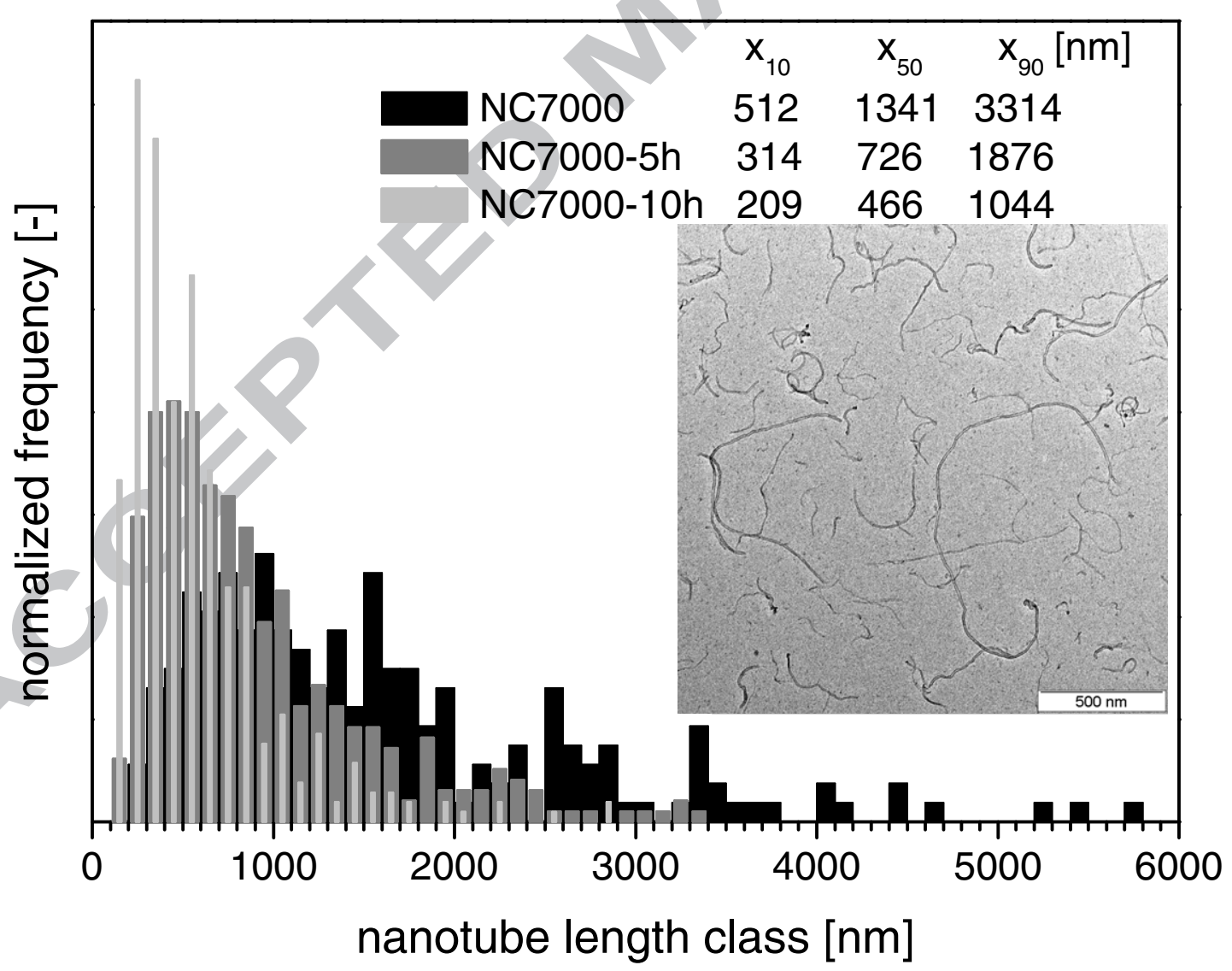

Fig. 3 


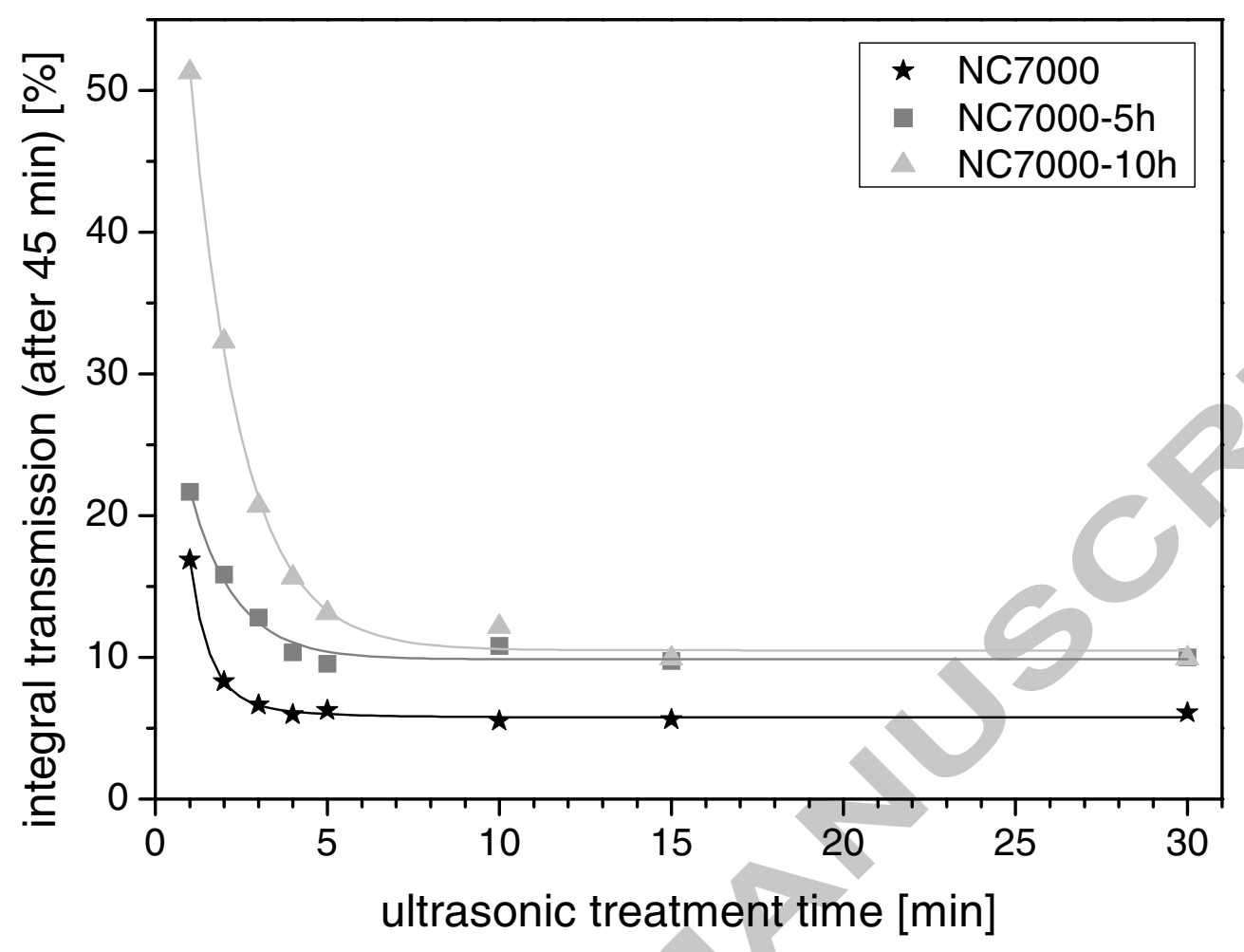

Fig. 4

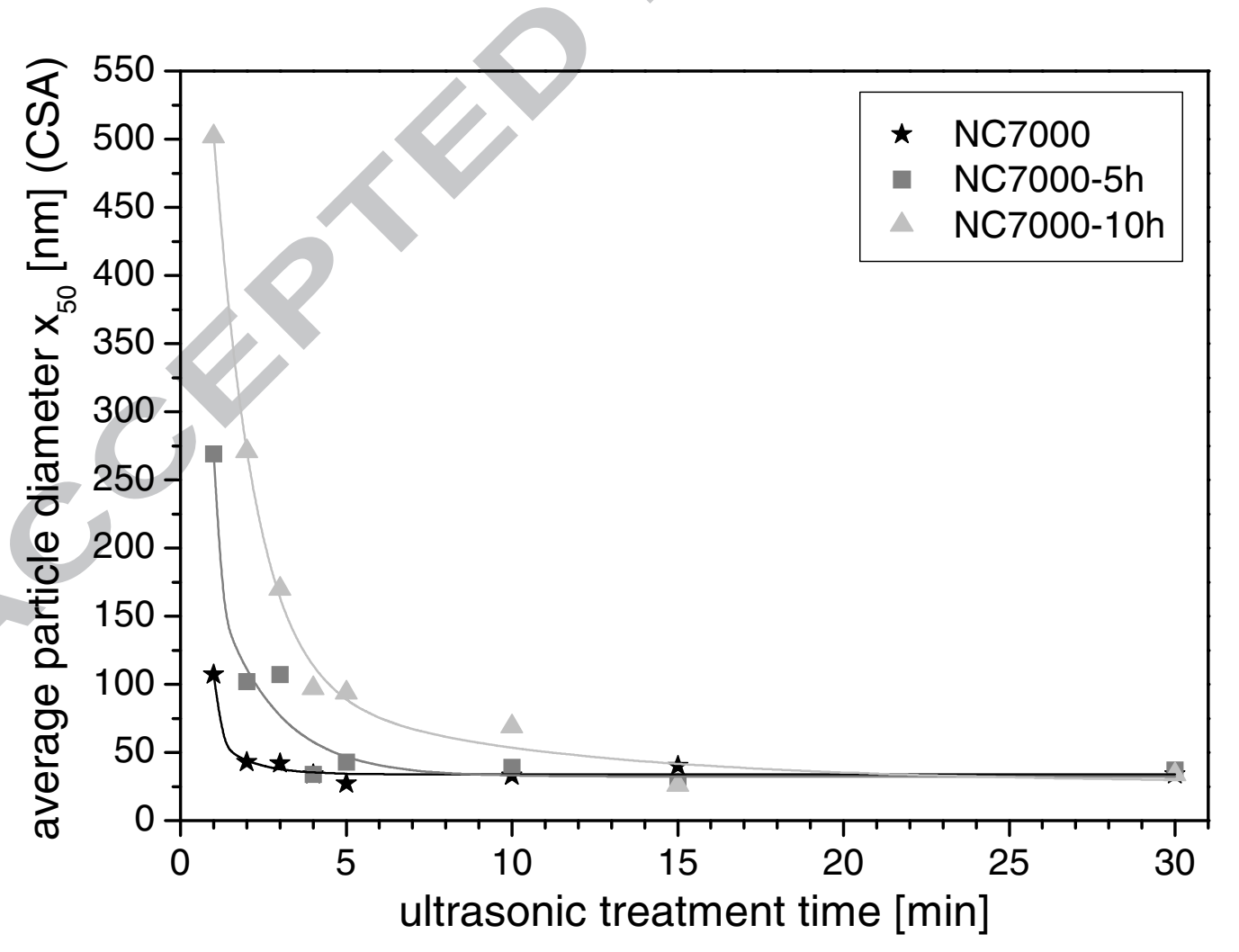

Fig. 5 


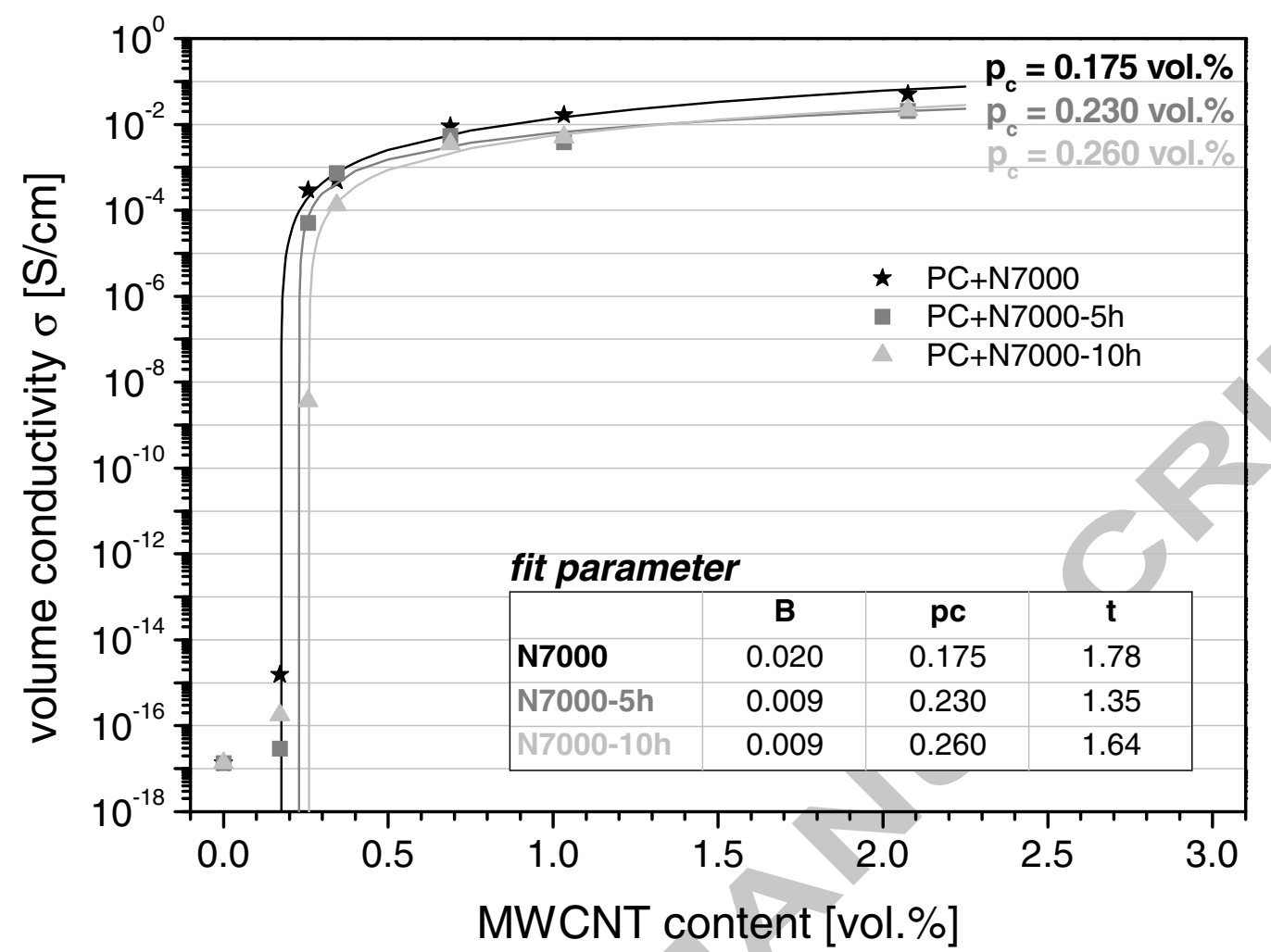

Fig. 6
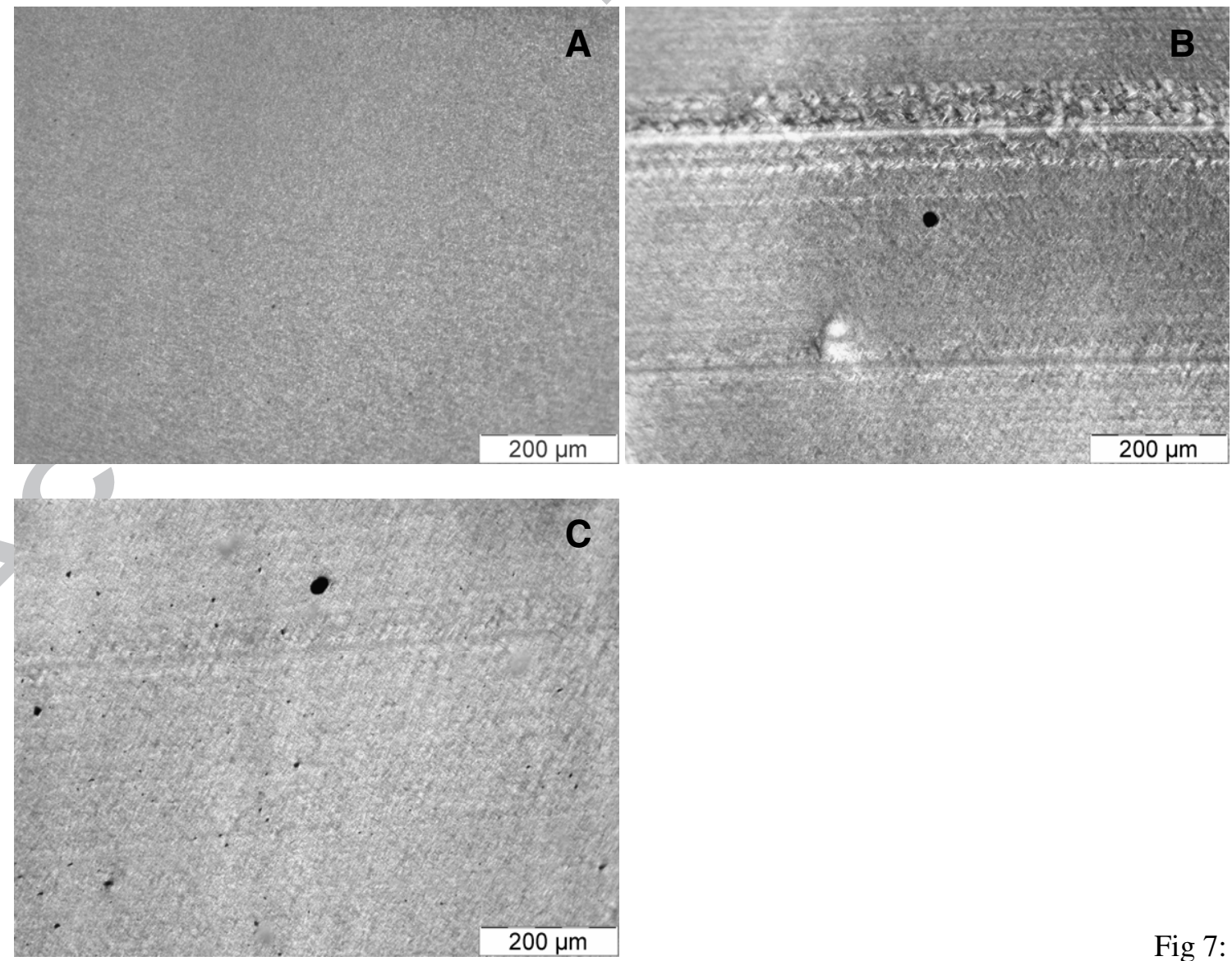

c

Fig 7: 


\section{ACCEPTED MANUSCRIPT}
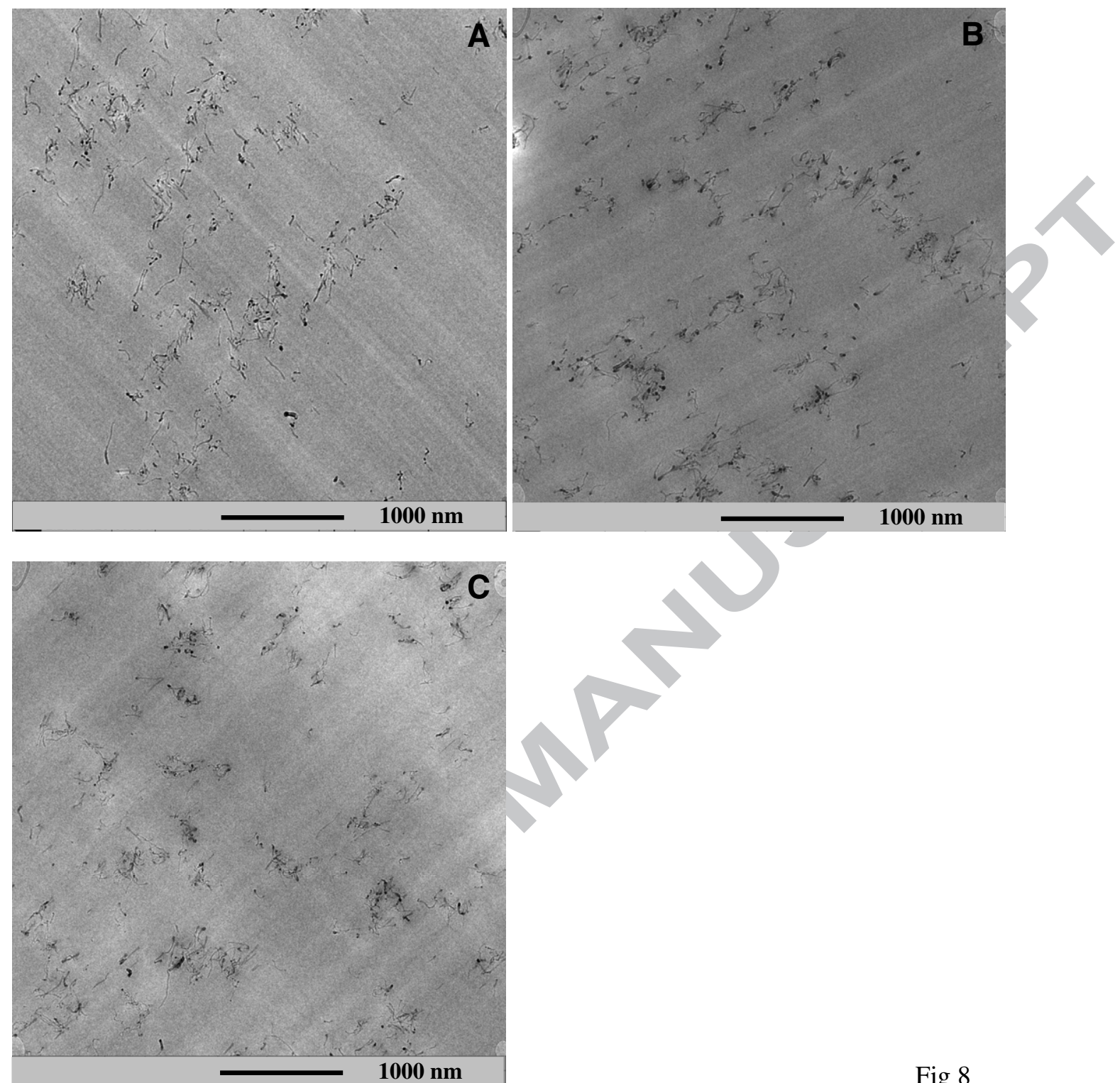

Fig 8 


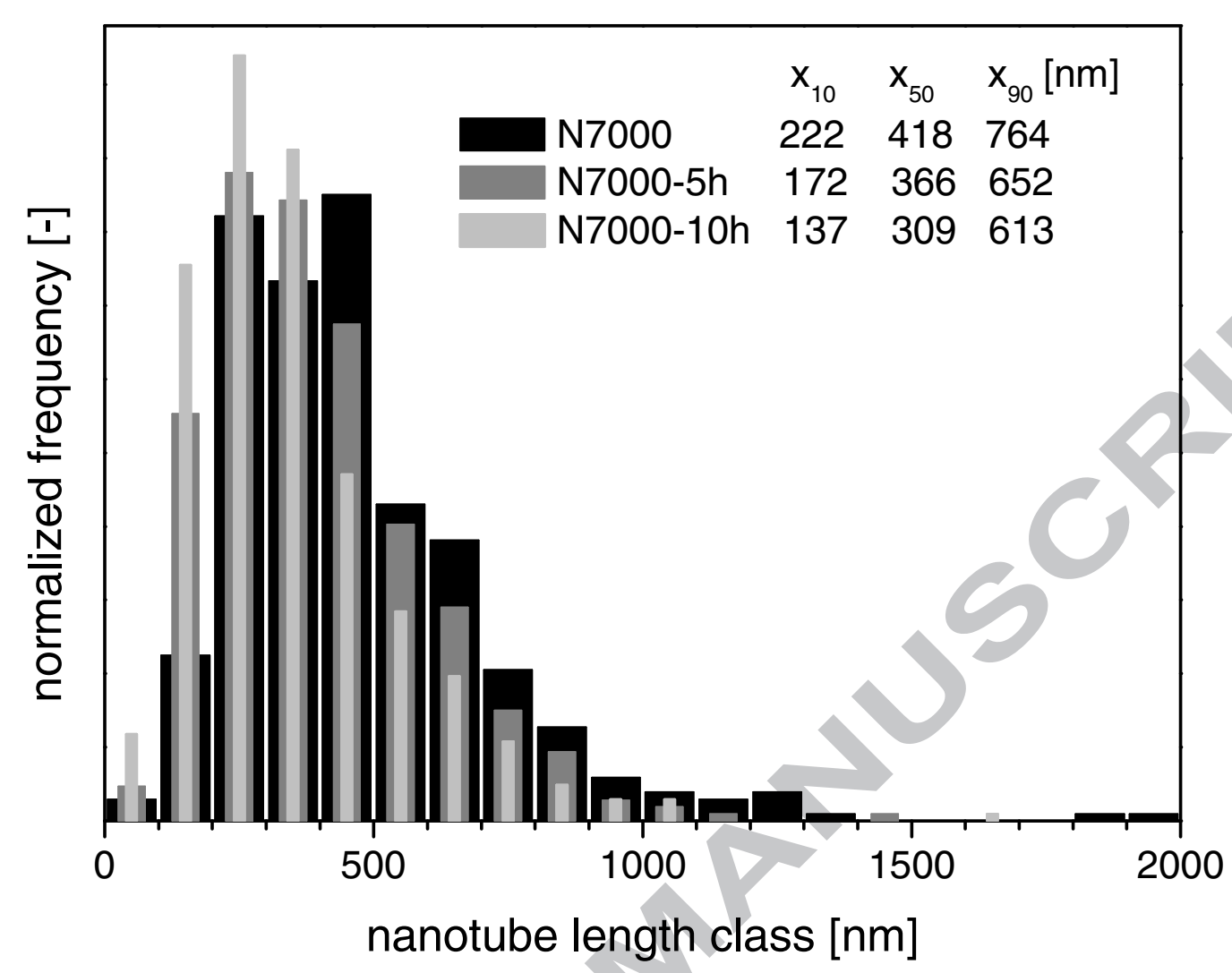

Fig. 9

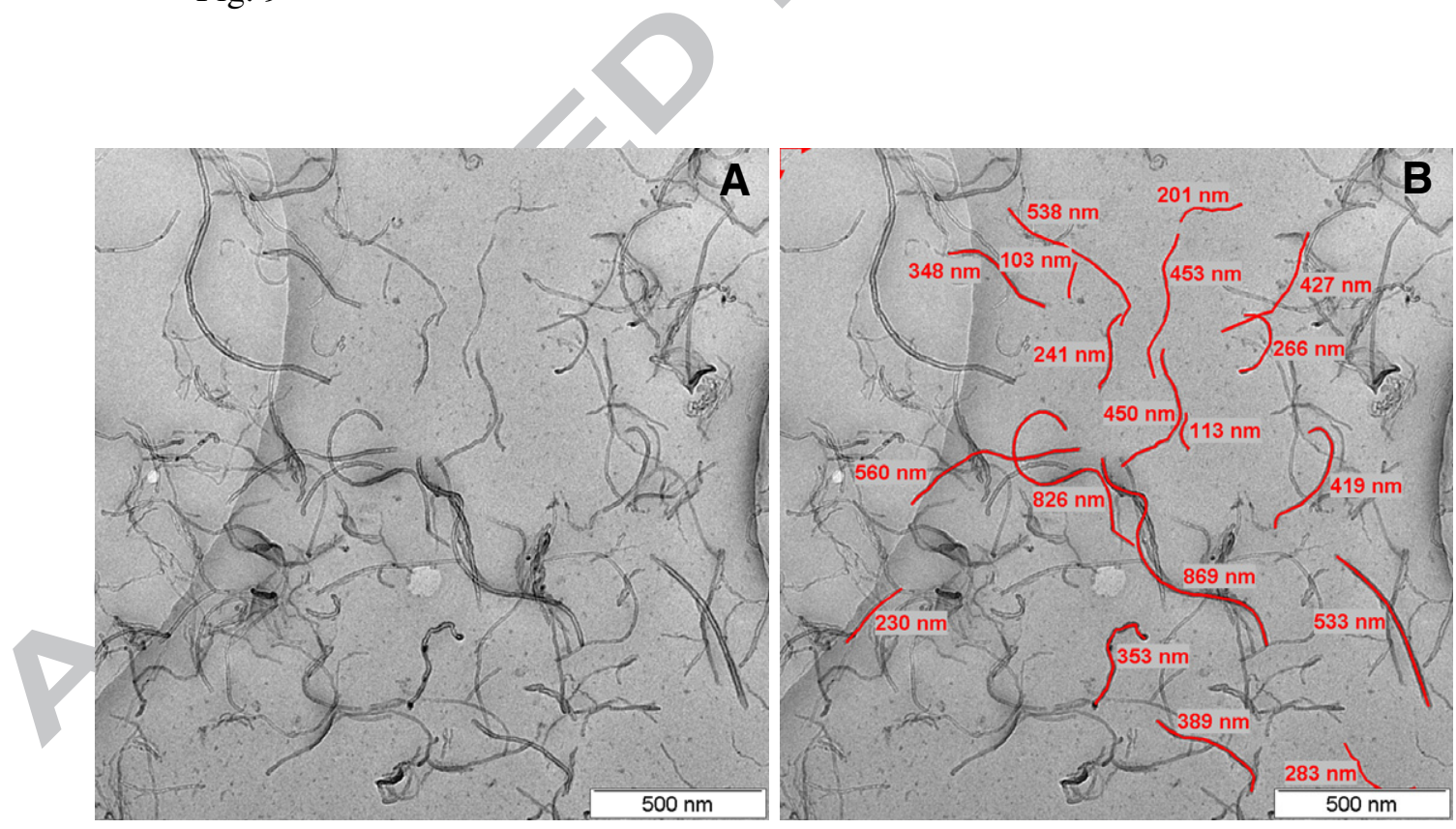

Fig 10 